\title{
Assessment of the anatomical position of point B and the relationship between point $B$ dose and the dose delivered to pelvic lymph nodes in CT-based high-dose-rate brachytherapy for uterine cervical cancer
}

\author{
Hideaki Matsukawa, BScl, Tomonari Sasaki, MD, PhD², Ryota Hirayama, MSc ${ }^{3}$, Taka-aki Hirose, BSc ${ }^{3}$, \\ Jun-ichi Fukunaga, $\mathrm{ASC}^{3}$ \\ 'Division of Medical Quantum Sciences, Department of Health Sciences, Graduate School of Medical Sciences, Kyushu University, Japan, \\ ${ }^{2}$ Department of Health Sciences, Graduate School of Medical Sciences, Kyushu University, Japan, ${ }^{3}$ Division of Radiology, Department of \\ Medical Technology, Kyushu University Hospital, Japan
}

\begin{abstract}
Purpose: To examine the anatomical position of point B and the relationship between the dose at point $\mathrm{B}$ and the dose delivered to the pelvic lymph nodes in computed tomography (CT)-based brachytherapy for cervical cancer.

Material and methods: Forty-nine cervical cancer patients were treated at Kyushu University Hospital. For all cases, planning CT images obtained after the applicator insertion were imported to an Oncentra Brachy (Elekta AB, Stockholm, Sweden), and points A (dose prescription, $6 \mathrm{~Gy}$ ) and points B were set according to the Manchester method. The pelvic lymph node regions (external iliac, internal iliac, and obturator) were contoured, and the anatomic positions of 98 points $B$ in 49 patients were examined. Dose volume histogram $(\mathrm{DVH})$ parameters $\left(\mathrm{D}_{100}, \mathrm{D}_{90}, \mathrm{D}_{50}, \mathrm{D}_{2 \mathrm{cc}}, \mathrm{D}_{1 \mathrm{cc},}\right.$ and $\mathrm{D}_{0.1 \mathrm{cc}}$ ) were calculated for each lymph node region and compared with the point $\mathrm{B}$ dose.

Results: The mean bilateral dose to point B was $1.70 \pm 0.18 \mathrm{~Gy}$, and $26(27 \%)$ of 98 points B were not located in any pelvic lymph node regions. The DVH analysis indicated a low degree of correlation overall, and all values were significantly different from point $\mathrm{B}$ doses $(p<0.05)$, except for $\mathrm{D}_{0.1 \mathrm{cc}}$ of the external iliac node $(p=0.0594)$ and $\mathrm{D}_{1 \mathrm{cc}}$ of the internal iliac node $(p=0.0711)$.

Conclusions: We investigated the anatomical location of point B in patients with cervical cancer who underwent brachytherapy, and the DVH analysis revealed that the point B dose was a poor surrogate for the dose delivered to the pelvic lymph nodes.

Key words: cervical cancer, brachytherapy, pelvis, lymph nodes, CT.

\section{Purpose}

Radiation therapy that is delivered by combining external beam radiotherapy (EBRT) and brachytherapy is a curative treatment option for uterine cervical cancer and is the standard therapy for a locally advanced disease $[1,2,3,4,5,6,7,8,9]$. Radiation therapy with a high-doserate (HDR) remote afterloading system for intracavitary brachytherapy is the standard treatment for uterine cervical cancer in Japan [10].

Two orthogonal X-ray images were used for conventional brachytherapy planning based on the Manchester method. The dose was prescribed to point A, which was defined as $2 \mathrm{~cm}$ lateral to the central uterine canal and $2 \mathrm{~cm}$ from the mucous membrane of the lateral fornix in the axis of the uterus. On the other hand, point B, which was designated as $5 \mathrm{~cm}$ from the midline at the level of point $\mathrm{A}$, represented the dose delivered to the pelvic lymph node, and was routinely recorded to calculate the integral dose to the pelvic sidewall delivered by EBRT and brachytherapy [11]. However, with the advent of three-dimensional (3D) image-guided brachytherapy (IGBT), the evaluation of dose distributions to assess the target coverage and to spar normal tissue has become
Address for correspondence: Tomonari Sasaki, MD, PhD, Department of Health Sciences, Graduate School of Medical Sciences, Kyushu University, 3-1-1 Maidashi, Higashi-ku, Fukuoka City, Fukuoka, 812-8582, Japan, phone: +81-92-642-6726, fax: +81-92-642-6674, $\bowtie$ e-mail: tomonari@hs.med.kyushu-u.ac.jp
Received: 25.08.2018

Accepted: 20.02.2019

Published: 29.04.2019 
possible $[12,13]$. Structures of interest can be more accurately evaluated with the dose volume histogram (DVH). The gynecologic GEC-ESTRO (Groupe Europeen de Curietherapie and the European Society for Radiotherapy and Oncology) Working Group has published guidelines on 3D image-based treatment planning for cervical cancer brachytherapy $[12,13]$. A previous study showed that while using IGBT, the clinical outcome presented an increase in local control and an approximately $20 \%$ decrease in toxicity compared with the 2D brachytherapy technique [14]. Derks et al. indicated that the 3D IGBT showed a trend for improved local control and overall survival compared to the 2D technique, and resulted in a decrease in observed toxicity from $17 \%$ (2D) to $12 \%$ (3D IGBT) [15]. Paul et al. reported a relative improvement in organs at risk (OAR) doses per HDR brachytherapy fraction by the volume-based HDR brachytherapy plan compared to the point A-based HDR brachytherapy plan, and described dosimetric advantages of using a volume-based HDR brachytherapy plan to create more conformal plans [16]. Moreover, Lee et al. examined the relationship between the point $B$ dose and the dose delivered to the pelvic nodal chains, and indicated that the point $B$ dose was a poor surrogate for the dose to specific nodal groups [11]. The relationship between the point $B$ dose and the dose delivered to the pelvic lymph node region has been reported in several studies [11,17], but these studies did not analyze the anatomical position of point $\mathrm{B}$ on a $3 \mathrm{D}$ view. In this study, we investigated the anatomical position of point $B$ and the relationship between the point $B$ dose and the dose delivered to the pelvic lymph nodes in computed tomography (CT)-based HDR brachytherapy for cervical cancer.

\section{Material and methods}

From February 2013 to January 2016, forty-nine patients with the International Federation of Gynecology and Obstetrics (FIGO) stage IB-IVB cervical cancer were treated at the Kyushu University Hospital. A tandem and ovoid applicator was used in 39 cases and a cylinder applicator was used in 10 cases. For all cases, CT images were obtained after applicator insertion for planning on a Plato treatment planning system (Elekta AB, Stockholm, Sweden). In our institutes, CT images were routinely obtained only after the initial applicator insertion to assess applicator position and dose distribution of the conventional 2D plan, before IGBT was introduced. This planning study is a retrospective analysis of the patient data when the conventional 2D plan was implemented. Therefore, we used the initial planning CT image per patient, not per fraction.

The initial planning $\mathrm{CT}$ images were imported to the treatment planning system Oncentra Brachy (Elekta AB, Stockholm, Sweden), and we reperformed the treatment planning again based on the Manchester method. A dose of $6 \mathrm{~Gy}$ was prescribed to point A with a standard dwell weight pattern [18], and point $B$ was set according to the Manchester method.

The pelvic lymph node regions (the external iliac, internal iliac, and obturator) were contoured as in the pre- vious reports $[11,19]$ by a radiation oncologist on the basis of CT images, using the pre-treatment MRI as a reference if necessary. The external iliac was contoured including the external iliac artery and vein, and a 7-mm vessel margin was set to encompass the medial, lateral, anterior, and posterior subgroups of the external iliac chain. This contour extended from the bottom of the sacroiliac joint superiorly to the top of the acetabulum inferiorly, along the iliopsoas muscle to include the lateral external iliac nodes and exclude the bones and muscles. The internal iliac node group included the internal iliac artery and vein. The contour began superiorly at the level of the sacroiliac joint and extended the roof of the acetabulum along the pelvic sidewall, like the external iliac chain. The obturator nodal contour extended from the fovea of the femoral head to the top of the acetabulum along the pelvic sidewall to exclude the muscle and OAR.

First, we examined where point $B$ was located among the following five volumes: the obturator node region, external iliac node region, internal iliac node region, inside of the pelvis, and outside of the pelvis. The inside of the pelvis was defined as an area in the pelvic cavity excluding the lymph node regions, and the outside of the pelvis was defined as the area of the pelvic bone and the region external to that. The DVH parameters were calculated for each pelvic lymph node group (left, right, and bilateral), and represented the doses received by $100 \%$, $90 \%$, and $50 \%$ of the volume $\left(\mathrm{D}_{100}, \mathrm{D}_{90}, \mathrm{D}_{50}\right)$ and the doses received by $2 \mathrm{cc}, 1 \mathrm{cc}$, and $0.1 \mathrm{cc}$ of tissue $\left(\mathrm{D}_{2 \mathrm{cc}}, \mathrm{D}_{1 \mathrm{cc}}, \mathrm{D}_{0.1 \mathrm{cc}}\right)$, respectively. The dose administered to point $\mathrm{B}$ was compared with the DVH parameters for each pelvic lymph node group. An ${ }^{192} \mathrm{Ir}$ source was used for the calculations in the treatment planning system. Additionally, DVH parameters for each lymph node region and point $\mathrm{B}$ dose were compared by applicator type (tandem and ovoid in 39 cases, tandem and cylinder in 10 cases).

For statistical analysis, JMP Pro13 software (SAS Institute Inc., Cary, NC, USA) was used, and the point B dose was compared with the DVH parameters for each lymph node group using a two-sided paired $t$-test. In order to examine the relationship between the point $\mathrm{B}$ dose and the DVH parameters, Pearson's correlation coefficients (CCs) were used. The comparison of DVH parameters and point $\mathrm{B}$ dose by applicator type was performed using a $t$-test. Statistical significance was accepted with a $p$ value $<0.05$.

\section{Results}

The patient and tumor characteristics are shown in Table 1. The median patient age was 62 years (range, 32-92 years) and of the 49 patients, 3 had FIGO stage IB, 3 had IIA, 17 had IIB, 2 had IIIA, 18 had IIIB, 2 had IVA, and 4 had IVB. The results for the anatomical position of point $\mathrm{B}$ are summarized in Table 2 and Figure 1. Seventy-two (73\%) of 98 points B (2 points per patient) were located in the pelvic lymph node regions. Fifty-two percent of bilateral points $B$ were in the obturator node region, and $21 \%$ were in the internal iliac node region. Twenty percent and $6 \%$ of points $B$ were inside and outside of the pelvis, respectively. No point was located in the external iliac 
Table 1. Patient and tumor characteristics

\begin{tabular}{lc} 
& Patients $(n=49)$ \\
\hline Age (years) & \\
\hline Median & 62 \\
\hline Range & $32-92$ \\
\hline FIGO stage & 3 \\
\hline IB & 3 \\
\hline IIA & 17 \\
\hline IIB & 2 \\
\hline III & 18 \\
\hline IIIB & 2 \\
\hline IVA & 4
\end{tabular}

FIGO - International Federation of Gynecology and Obstetrics

chain in this study (49 patients). Figures 2 and 3 shows the pelvic lymph node volumes and the point $B$ in relation to the dose distribution in a typical patient, where the point $B$ is located in the pelvic lymph node region and in a typical patient, where the point $B$ is not located in the pelvic lymph node region, respectively.

For 49 applications, the mean bilateral dose to point B was $1.70 \pm 0.18 \mathrm{~Gy}$ (right dose, $1.64 \pm 0.34 \mathrm{~Gy}$; left dose,
Table 2. The anatomical location of point B for 49 cervical cancer patients

\begin{tabular}{lcc} 
& Point B (right) & Point B (left) \\
\hline Obturator node region & $53.1 \%(26 / 49)$ & $51.0 \%(25 / 49)$ \\
\hline External iliac node region & $0 \%(0 / 49)$ & $0 \%(0 / 49)$ \\
\hline Internal iliac node region & $20.4 \%(10 / 49)$ & $22.4 \%(11 / 49)$ \\
\hline Inside of the pelvis & $22.4 \%(11 / 49)$ & $18.4 \%(9 / 49)$ \\
\hline Outside of the pelvis & $4.1 \%(2 / 49)$ & $8.2 \%(4 / 49)$
\end{tabular}

$1.76 \pm 0.35 \mathrm{~Gy})$. The DVH parameters $\left(\mathrm{D}_{100}, \mathrm{D}_{90}, \mathrm{D}_{50}, \mathrm{D}_{2 \mathrm{cc}}\right.$ $\mathrm{D}_{1 \mathrm{cc}}$ and $\left.\mathrm{D}_{0.1 \mathrm{cc}}\right)$ for the pelvic lymph node groups and the statistical analysis results are shown in Table 3. The CCs were less than 0.7 , except for $\mathrm{D}_{50}$ of the obturator lymph node group (CC, 0.73) (bilateral), and the results indicated a low degree of correlation overall. All parameters of the obturator, $\mathrm{D}_{100}, \mathrm{D}_{90}, \mathrm{D}_{50}, \mathrm{D}_{2 \mathrm{cc}}$ and $\mathrm{D}_{1 \mathrm{cc}}$ of the external iliac node and $\mathrm{D}_{100}, \mathrm{D}_{90}, \mathrm{D}_{50}, \mathrm{D}_{2 \mathrm{cc}}$ and $\mathrm{D}_{0.1 \mathrm{cc}}$ of the internal iliac node were significantly different from the point $\mathrm{B}$ doses. However, $\mathrm{D}_{0.1 \mathrm{cc}}$ of the external iliac node group and $\mathrm{D}_{1 \mathrm{cc}}$ of the internal iliac node group were not statistically different from the point $\mathrm{B}$ doses. For the obturator group, the mean $\mathrm{D}_{50}$ was $1.47 \pm 0.28 \mathrm{~Gy}$ ( $t$-test,

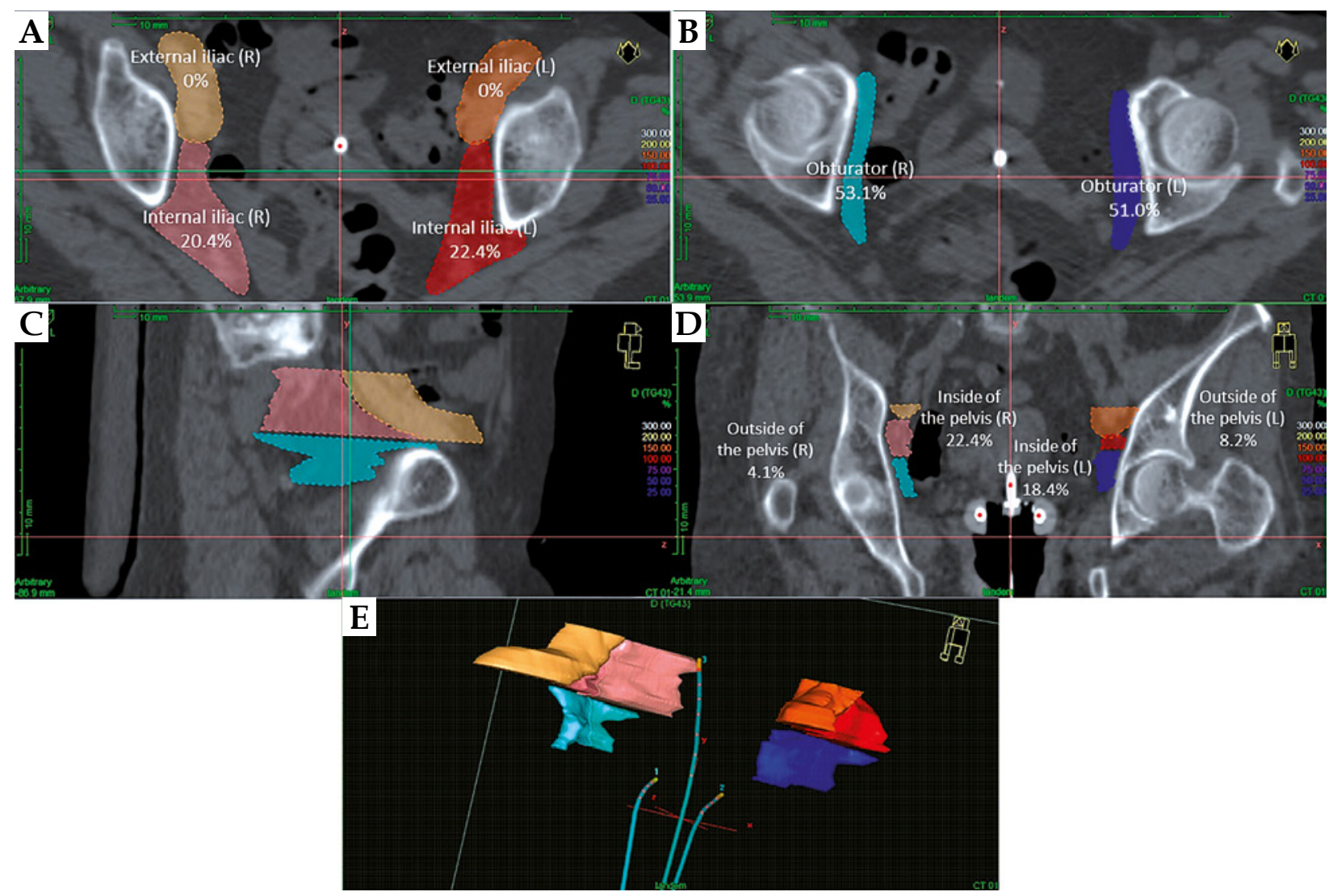

Pelvic lymph node regions: obturator in blue, external iliac node in orange, and internal iliac node in red. $R$-right, $L$ - left

Fig. 1. Contoured pelvic lymph node regions and the percentage values of the anatomical location of point $\mathrm{B}$ on $\mathrm{CT}$ image. A) The axial plane, on which the external and internal iliac nodes are observed; B) The axial plane, on which the obturator is observed; C) The sagittal plane; D) The coronal plane; E) 3D view 


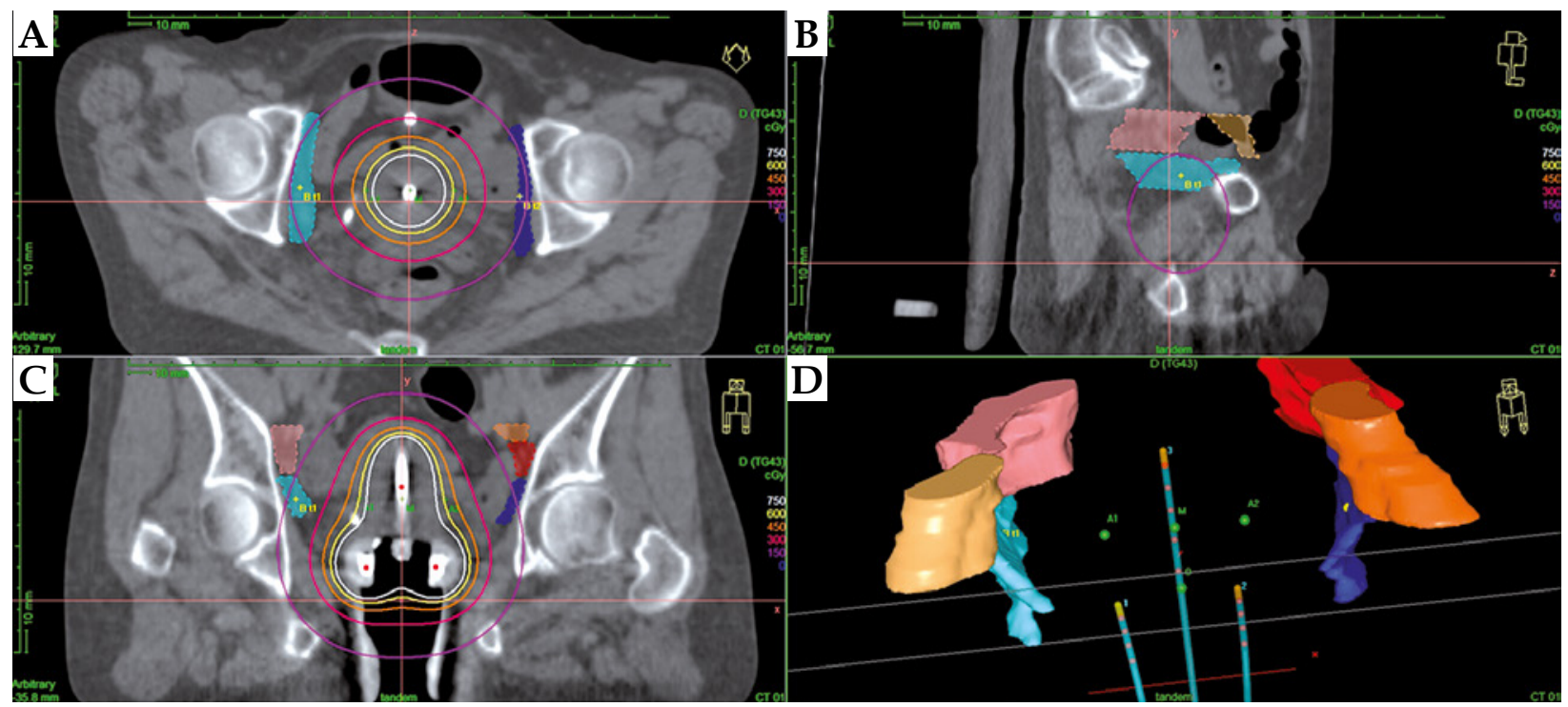

Pelvic lymph node regions: obturator in blue, external iliac node in orange, and internal iliac node in red. Points: point $B$ in yellow, point $A$ in green.

Fig. 2. The pelvic lymph node volumes and point B in relation to the dose distribution, in a typical patient, where point $B$ is located in the pelvic lymph node region. A) Axial plane; B) Sagittal plane; C) Coronal plane; D) 3D view

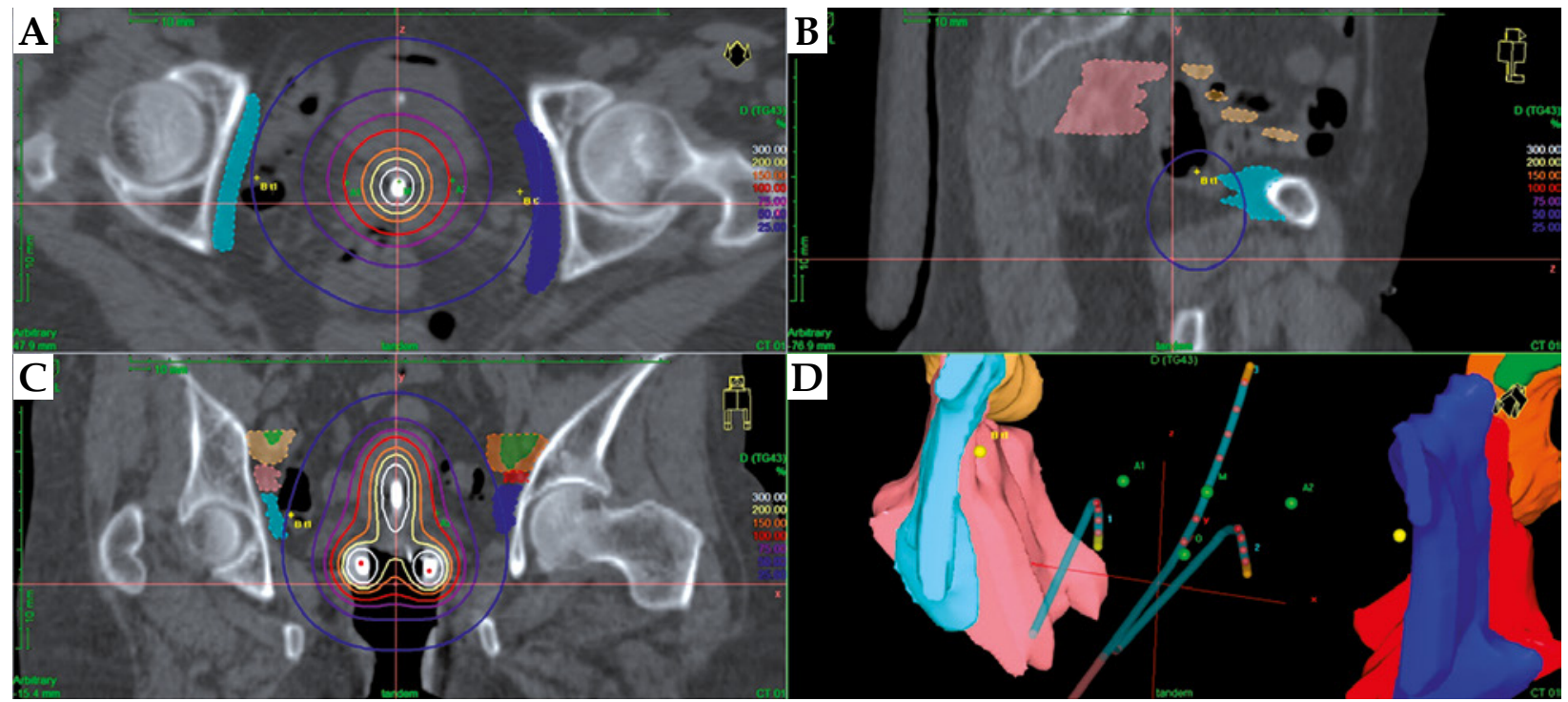

Pelvic lymph node regions: obturator in blue, external iliac node in orange, and internal iliac node in red. Points: point $B$ in yellow, point $A$ in green.

Fig. 3. The pelvic lymph node volumes and the point $B$ in relation to the dose distribution, in a typical patient, where the point $B$ is not located in the pelvic lymph node region. A) Axial plane; B) Sagittal plane; C) Coronal plane, D) 3D view

$p<0.0001$ ), and the CC for $\mathrm{D}_{50}$ of the obturator was only slightly more than 0.7 (CC, $0.73, p<0.0001)$. However, all DVH parameters were significantly different from the point B dose. The DVH parameter of the obturator that most closely represented the point $B$ dose was $D_{2 c^{\prime}}$ and the mean value was $1.81 \pm 0.35 \mathrm{~Gy}(t$-test, $p=0.0089)$ with a CC of $0.68(p<0.0001)$. In this study, we found that the point $\mathrm{B}$ doses were not adequate surrogates for the DVH parameters of the obturator group. For the external iliac chain, the mean $\mathrm{D}_{0.1 \mathrm{cc}}$ was $1.60 \pm 0.37 \mathrm{~Gy}(p=0.0594)$, and the CC was $0.32(p=0.0236)$ (Figure 4$)$. The $\mathrm{D}_{100}, \mathrm{D}_{90}, \mathrm{D}_{50}$, $\mathrm{D}_{2 \mathrm{cc}}$ and $\mathrm{D}_{1 \mathrm{cc}}$ were statistically different from the point $\mathrm{B}$ dose $(t$-test, $p<0.0001)$. For the internal iliac chain, the mean $\mathrm{D}_{1 \text { cc }}$ was $1.60 \pm 0.39 \mathrm{~Gy}$ ( $t$-test, $p=0.0711$ ), and this parameter alone did not indicate a significant difference. The $\mathrm{CC}$ of $\mathrm{D}_{1 \mathrm{cc}}$ was $0.33\left(p=0.0223\right.$ ) (Figure 5). The $\mathrm{D}_{100}$, $\mathrm{D}_{90}, \mathrm{D}_{50}, \mathrm{D}_{2 \mathrm{cc}}$, and $\mathrm{D}_{0.1 \mathrm{cc}}$ for the internal iliac chain were statistically different from the point $B$ dose. The mean uterine length and separation between ovoids were $61.6 \mathrm{~mm}$ (range, $41.1-83.4 \mathrm{~mm}$ ) and $32.1 \mathrm{~mm}$ (range, 22.6-42.1 mm), respectively. The correlation between these values and the doses delivered to pelvic lymph node regions was analyzed. The separation between ovoids did not correlate with the doses to pelvic lymph nodes significantly; 
Table 3. The DVH parameters $\left(D_{100}, D_{90}, D_{50}, D_{2 c c}, D_{1 c c}\right.$, and $\left.D_{0.1 c c}\right)$ for pelvic lymph node groups and correlation coefficients between these parameters and point $\mathrm{B}$ dose

\begin{tabular}{|c|c|c|c|c|c|}
\hline & $\begin{array}{c}\text { Bilateral mean } \\
\text { dose }(G y)\end{array}$ & $\mathrm{CC}^{1}$ & $p$ value $^{2}$ & $t$-test ${ }^{3}$ & $\begin{array}{c}\text { Percentage } \\
(\%)\end{array}$ \\
\hline \multicolumn{6}{|c|}{ Obturator node group } \\
\hline$D_{100^{5}}$ & $0.65 \pm 0.17$ & 0.60 & $<0.0001$ & $<0.0001$ & $10.8 \pm 2.87$ \\
\hline$D_{90}{ }^{6}$ & $1.07 \pm 0.22$ & 0.66 & $<0.0001$ & $<0.0001$ & $17.8 \pm 3.74$ \\
\hline$D_{50}{ }^{7}$ & $1.47 \pm 0.28$ & 0.73 & $<0.0001$ & $<0.0001$ & $24.5 \pm 4.61$ \\
\hline$D_{2 c c}{ }^{8}$ & $1.81 \pm 0.35$ & 0.68 & $<0.0001$ & 0.0089 & $30.1 \pm 5.87$ \\
\hline $\mathrm{D}_{1 c c}{ }^{9}$ & $1.95 \pm 0.38$ & 0.66 & $<0.0001$ & $<0.0001$ & $32.4 \pm 6.37$ \\
\hline $\mathrm{D}_{0.1 c c}{ }^{10}$ & $2.27 \pm 0.45$ & 0.64 & $<0.0001$ & $<0.0001$ & $37.9 \pm 7.56$ \\
\hline \multicolumn{6}{|c|}{ External iliac nodes } \\
\hline $\mathrm{D}_{100}$ & $0.29 \pm 0.10$ & 0.55 & $<0.0001$ & $<0.0001$ & $4.80 \pm 1.62$ \\
\hline$D_{90}$ & $0.59 \pm 0.15$ & 0.56 & $<0.0001$ & $<0.0001$ & $9.82 \pm 2.55$ \\
\hline$D_{50}$ & $0.90 \pm 0.22$ & 0.52 & 0.0001 & $<0.0001$ & $14.9 \pm 3.66$ \\
\hline $\mathrm{D}_{2 c c}$ & $1.24 \pm 0.31$ & 0.41 & 0.0037 & $<0.0001$ & $20.7 \pm 5.16$ \\
\hline $\mathrm{D}_{1 c c}$ & $1.35 \pm 0.34$ & 0.38 & 0.0069 & $<0.0001$ & $22.5 \pm 5.59$ \\
\hline $\mathrm{D}_{0.1 c c}$ & $1.60 \pm 0.37$ & 0.32 & 0.0236 & 0.0594 & $26.7 \pm 6.24$ \\
\hline \multicolumn{6}{|c|}{ Internal iliac nodes } \\
\hline$D_{100}$ & $0.49 \pm 0.14$ & 0.47 & 0.0006 & $<0.0001$ & $8.22 \pm 2.31$ \\
\hline $\mathrm{D}_{90}$ & $0.81 \pm 0.19$ & 0.43 & 0.0021 & $<0.0001$ & $13.5 \pm 3.19$ \\
\hline$D_{50}$ & $1.08 \pm 0.25$ & 0.39 & 0.0054 & $<0.0001$ & $18.0 \pm 4.11$ \\
\hline $\mathrm{D}_{2 c c}$ & $1.49 \pm 0.36$ & 0.33 & 0.0198 & $<0.0001$ & $24.8 \pm 6.04$ \\
\hline$\overline{D_{1 c c}}$ & $1.60 \pm 0.39$ & 0.33 & 0.0223 & 0.0711 & $26.7 \pm 6.49$ \\
\hline $\mathrm{D}_{0.1 c c}$ & $1.89 \pm 0.45$ & 0.29 & 0.0396 & 0.0043 & $31.5 \pm 7.55$ \\
\hline Point B dose & $1.70 \pm 0.18$ & & & & $28.3 \pm 2.98$ \\
\hline
\end{tabular}

${ }^{1} \mathrm{CC}$ - correlation coefficient; ${ }^{2} p$ value - $p$ value calculated by Pearson's correlation coefficients; ${ }^{3} t$-test- $p$ value calculated by a two sided paired $t$-test; ${ }^{4}$ Percentage - percentage of the prescription dose (6 Gy); ${ }^{5} D_{100}$ - dose to $100 \%$ of the volume; ${ }^{6} D_{90}$-dose to $90 \%$ of the volume; ${ }^{7} D_{50}-$ dose to $50 \%$ of the volume; ${ }^{8} D_{2 c c}-$ dose to $2 \mathrm{cc}$ of the volume; ${ }^{9} D_{1 c c}$ - dose to $1 \mathrm{cc}$ of the volume; ${ }^{10} D_{0.1 c c}$ - dose to $0.1 \mathrm{cc}$ of the volume

however, we found the positive significant correlation between the uterine length and the doses to the external iliac node.

In addition, Table 4 shows the comparison of DVH parameters for each lymph node region and point $\mathrm{B}$ dose by applicator type, including 39 tandem and ovoid and 10 tandem and cylinder applicators. The dose to the obturator nodes was lower 0.27-0.67 Gy for the tandem and cylinder applicator when compared by a $t$-test, although there was not such a significant difference for the external iliac nodes and the internal iliac nodes. We also analyzed the correlation between point $\mathrm{B}$ dose and the dose delivered to the pelvic lymph node regions by applicator type. However, we could not find a significant correlation between point $B$ dose and the dose delivered to the pelvic lymph node regions in tandem and cylinder applicator cases, probably because sample size of the cylinder applicator was small (Table 5).

\section{Discussion}

The present study was carried out to evaluate the anatomical position of point $\mathrm{B}$, and the relationship between the point $\mathrm{B}$ dose and the dose delivered to the pelvic lymph nodes in HDR brachytherapy for uterine cervical cancer according to the Manchester method. We found that $27 \%$ of points $B$ were not located in any pelvic lymph node regions. For DVH analysis, the CCs were less than 0.7 , except for the $\mathrm{D}_{50}$ of the obturator group. As in the previous study [11], we indicated that the DVH parameters were statistically different from the point B dose, and the pelvic lymph node contours defined the nodal dose more accurately than did point $\mathrm{B}$. The two DVH parameters, i.e., the $\mathrm{D}_{0.1 \mathrm{cc}}$ of the external iliac chain and the $\mathrm{D}_{1 \mathrm{cc}}$ of the internal iliac chain, were not statistically different from the point $\mathrm{B}$ dose; however, the low $\mathrm{CC}$ of these parameters suggests that the point $B$ dose was not a clinically significant surrogate for the dose delivered to the 


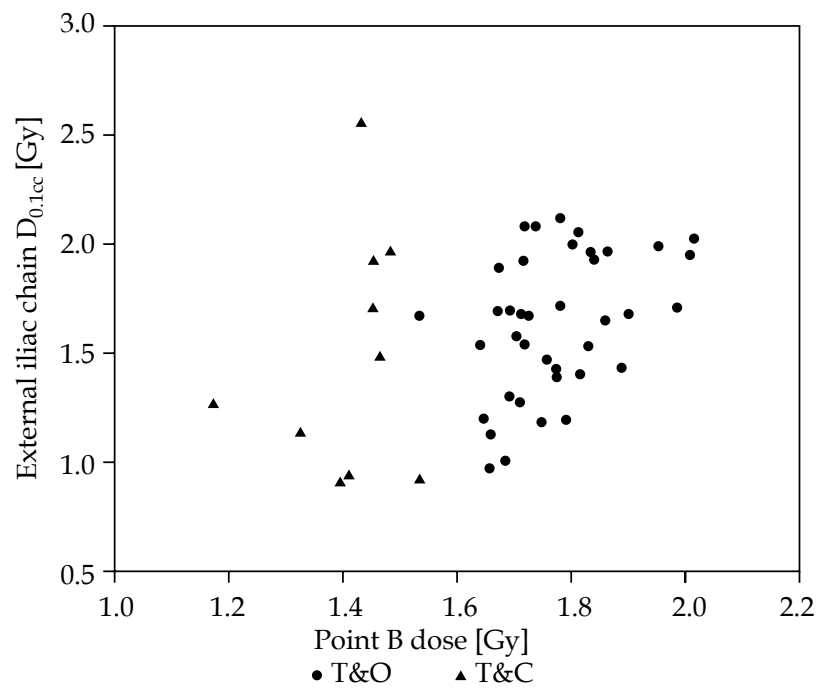

$T \& O$ - tandem and ovoid applicator (circle point), T\&C - tandem and cylinder applicator (triangle point)

Fig. 4. Correlation between the point $\mathrm{B}$ dose and $\mathrm{D}_{0.1 \mathrm{cc}}$ of the external iliac chain (CC, 0.32). The scatter-plot points were categorized into two forms by the applicator type

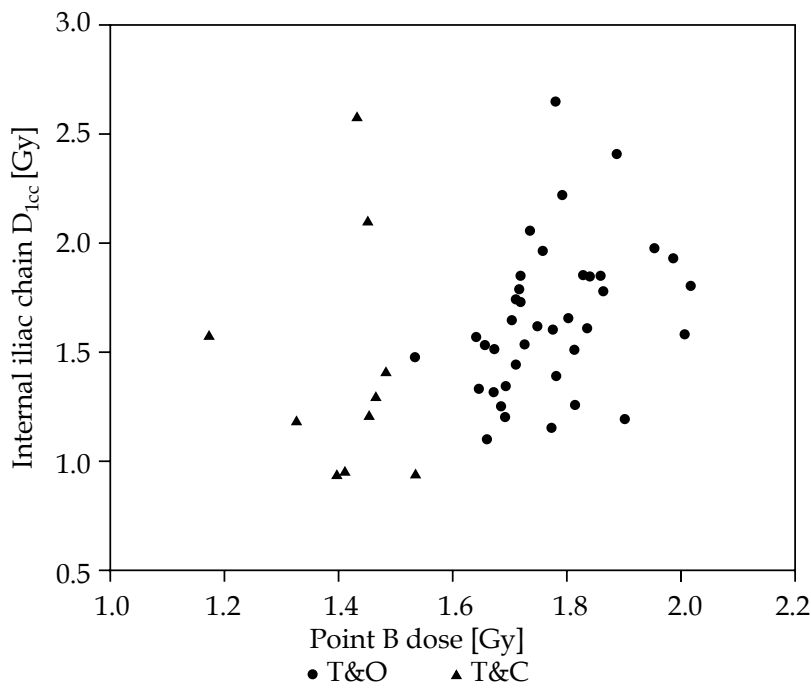

$T \& O$ - tandem and ovoid applicator (circle point), T\&C - tandem and cylinder applicator (triangle point)

Fig. 5. Correlation between the point $B$ dose and $D_{1 c c}$ of the internal iliac chain $(C C, 0.33)$. The scatter-plot points were categorized into two forms by the applicator type

Table 4. Comparison of DVH parameters for each nodal region and point $B$ dose by applicator type
$\mathrm{T} \& \mathrm{O}^{1}$ mean dose (Gy)
$T \& C^{2}$ mean dose (Gy)
$\Delta^{3}(\mathrm{~Gy})$
$t$-test ${ }^{4}$

$n=39$ $n=10$

\begin{tabular}{|c|c|c|c|c|}
\hline \multicolumn{5}{|c|}{ Obturator node group } \\
\hline $\mathrm{D}_{100^{5}}$ & $0.70 \pm 0.14$ & $0.43 \pm 0.11$ & 0.27 & $<0.0001$ \\
\hline$D_{90}{ }^{6}$ & $1.15 \pm 0.17$ & $0.76 \pm 0.13$ & 0.39 & $<0.0001$ \\
\hline $\mathrm{D}_{50}{ }^{7}$ & $1.57 \pm 0.20$ & $1.08 \pm 0.18$ & 0.49 & $<0.0001$ \\
\hline $\mathrm{D}_{2 c c^{8}}$ & $1.92 \pm 0.27$ & $1.36 \pm 0.28$ & 0.56 & $<0.0001$ \\
\hline $\mathrm{D}_{1 c c}{ }^{9}$ & $2.07 \pm 0.30$ & $1.48 \pm 0.29$ & 0.59 & $<0.0001$ \\
\hline$D_{0.1 c c}{ }^{10}$ & $2.41 \pm 0.38$ & $1.74 \pm 0.33$ & 0.67 & $<0.0001$ \\
\hline \multicolumn{5}{|c|}{ External iliac nodes } \\
\hline$D_{100}$ & $0.31 \pm 0.09$ & $0.20 \pm 0.07$ & 0.11 & 0.0005 \\
\hline $\mathrm{D}_{90}$ & $0.62 \pm 0.13$ & $0.45 \pm 0.15$ & 0.17 & 0.001 \\
\hline $\mathrm{D}_{50}$ & $0.94 \pm 0.20$ & $0.74 \pm 0.24$ & 0.20 & 0.0097 \\
\hline$D_{2 c c}$ & $1.28 \pm 0.27$ & $1.09 \pm 0.39$ & 0.19 & 0.0753 \\
\hline$D_{1 c c}$ & $1.39 \pm 0.29$ & $1.20 \pm 0.43$ & 0.19 & 0.1231 \\
\hline $\mathrm{D}_{0.1 c c}$ & $1.63 \pm 0.32$ & $1.48 \pm 0.52$ & 0.15 & 0.2822 \\
\hline \multicolumn{5}{|c|}{ Internal iliac nodes } \\
\hline$D_{100}$ & $0.52 \pm 0.12$ & $0.40 \pm 0.17$ & 0.12 & 0.0228 \\
\hline $\mathrm{D}_{90}$ & $0.84 \pm 0.16$ & $0.70 \pm 0.24$ & 0.14 & 0.0433 \\
\hline$D_{50}$ & $1.11 \pm 0.21$ & $0.96 \pm 0.32$ & 0.15 & 0.0838 \\
\hline$D_{2 c c}$ & $1.53 \pm 0.31$ & $1.32 \pm 0.48$ & 0.21 & 0.0985 \\
\hline $\mathrm{D}_{1 \mathrm{cc}}$ & $1.65 \pm 0.34$ & $1.42 \pm 0.51$ & 0.23 & 0.1038 \\
\hline $\mathrm{D}_{0.1 c c}$ & $1.94 \pm 0.39$ & $1.71 \pm 0.60$ & 0.23 & 0.162 \\
\hline Point B dose & $1.77 \pm 0.11$ & $1.41 \pm 0.10$ & 0.36 & $<0.0001$ \\
\hline
\end{tabular}

${ }^{1} T \& O$ - tandem and ovoid applicator. ${ }^{2} T \& C$ - tandem and cylinder applicator. ${ }^{3} \Delta$ - dose difference between T\&O and T\&C (T\&O mean dose - T\&C mean dose); ${ }^{4} t$ test - $p$ value calculated by a t-test; ${ }^{5} D_{100}$-dose to $100 \%$ of the volume; ${ }^{6} D_{90}$-dose to $90 \%$ of the volume; ${ }^{7} D_{50}-$ dose to $50 \%$ of the volume; ${ }^{8} D_{2 c c}-$ dose to $2 \mathrm{cc}$ of the volume; ${ }^{9} D_{1 \mathrm{cc}}$ - dose to $1 \mathrm{cc}$ of the volume; ${ }^{10} \mathrm{D}_{0.1 \mathrm{cc}}$ - dose to $0.1 \mathrm{cc}$ of the volume 
Table 5. The DVH parameters $\left(D_{100}, D_{90}, D_{50}, D_{2 c c}, D_{1 c c}\right.$, and $\left.D_{0.1 c c}\right)$ for pelvic lymph node groups, and correlation coefficients between these parameters and point $\mathrm{B}$ dose by applicator type

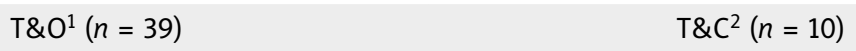

\begin{tabular}{|c|c|c|c|c|c|c|c|c|}
\hline & & & & & & & & \\
\hline & $\begin{array}{l}\text { Bilateral } \\
\text { mean dose } \\
\text { (Gy) }\end{array}$ & $\mathrm{CC}^{3}$ & $p$ value $^{4}$ & $t$-test $^{5}$ & $\begin{array}{c}\text { Bilateral } \\
\text { mean dose } \\
(G y)\end{array}$ & CC & $p$ value & $t$-test \\
\hline \multicolumn{9}{|c|}{ Obturator node group } \\
\hline$D_{100}{ }^{6}$ & $0.70 \pm 0.14$ & 0.19 & 0.2584 & $<0.0001$ & $0.43 \pm 0.11$ & 0.16 & 0.6495 & $<0.0001$ \\
\hline $\mathrm{D}_{90}{ }^{7}$ & $1.15 \pm 0.17$ & 0.21 & 0.2092 & $<0.0001$ & $0.76 \pm 0.13$ & 0.34 & 0.3364 & $<0.0001$ \\
\hline$D_{50}{ }^{8}$ & $1.57 \pm 0.20$ & 0.33 & 0.0404 & $<0.0001$ & $1.08 \pm 0.18$ & 0.50 & 0.1437 & 0.0001 \\
\hline$D_{2 c c}{ }^{9}$ & $1.92 \pm 0.27$ & 0.33 & 0.0418 & 0.0013 & $1.36 \pm 0.28$ & 0.48 & 0.1606 & 0.5475 \\
\hline$D_{1 c c}{ }^{10}$ & $2.07 \pm 0.30$ & 0.31 & 0.0541 & $<0.0001$ & $1.48 \pm 0.29$ & 0.51 & 0.1343 & 0.4922 \\
\hline$D_{0.1 c c}^{11}$ & $2.41 \pm 0.38$ & 0.31 & 0.0576 & $<0.0001$ & $1.74 \pm 0.33$ & 0.52 & 0.1196 & 0.0078 \\
\hline \multicolumn{9}{|c|}{ External iliac nodes } \\
\hline$D_{100}$ & $0.31 \pm 0.09$ & 0.32 & 0.0461 & $<0.0001$ & $0.20 \pm 0.07$ & 0.26 & 0.4598 & $<0.0001$ \\
\hline $\mathrm{D}_{90}$ & $0.62 \pm 0.13$ & 0.40 & 0.0106 & $<0.0001$ & $0.45 \pm 0.15$ & 0.24 & 0.5046 & $<0.0001$ \\
\hline$D_{50}$ & $0.94 \pm 0.20$ & 0.46 & 0.0034 & $<0.0001$ & $0.74 \pm 0.24$ & 0.22 & 0.5321 & $<0.0001$ \\
\hline $\mathrm{D}_{2 \mathrm{cc}}$ & $1.28 \pm 0.27$ & 0.41 & 0.0095 & $<0.0001$ & $1.09 \pm 0.39$ & 0.20 & 0.5725 & 0.0288 \\
\hline$D_{1 c c}$ & $1.39 \pm 0.29$ & 0.41 & 0.0093 & $<0.0001$ & $1.20 \pm 0.43$ & 0.21 & 0.5644 & 0.1676 \\
\hline $\mathrm{D}_{0.1 \mathrm{cc}}$ & $1.63 \pm 0.32$ & 0.41 & 0.0104 & 0.0044 & $1.48 \pm 0.52$ & 0.21 & 0.5572 & 0.686 \\
\hline \multicolumn{9}{|c|}{ Internal iliac nodes } \\
\hline$D_{100}$ & $0.52 \pm 0.12$ & 0.51 & 0.001 & $<0.0001$ & $0.40 \pm 0.17$ & -0.0045 & 0.9902 & $<0.0001$ \\
\hline$D_{90}$ & $0.84 \pm 0.16$ & 0.46 & 0.0031 & $<0.0001$ & $0.70 \pm 0.24$ & 0.040 & 0.9132 & $<0.0001$ \\
\hline$D_{50}$ & $1.11 \pm 0.21$ & 0.46 & 0.0029 & $<0.0001$ & $0.96 \pm 0.32$ & -0.00021 & 0.9995 & 0.0028 \\
\hline$D_{2 c c}$ & $1.53 \pm 0.31$ & 0.36 & 0.024 & $<0.0001$ & $1.32 \pm 0.48$ & -0.060 & 0.8699 & 0.5741 \\
\hline $\mathrm{D}_{1 \mathrm{icc}}$ & $1.65 \pm 0.34$ & 0.36 & 0.026 & 0.0176 & $1.42 \pm 0.51$ & -0.071 & 0.8453 & 0.9691 \\
\hline$D_{0.1 c c}$ & $1.94 \pm 0.39$ & 0.35 & 0.031 & 0.0109 & $1.71 \pm 0.60$ & -0.084 & 0.8175 & 0.1859 \\
\hline Point B dose & $1.77 \pm 0.11$ & & & & $1.41 \pm 0.10$ & & & \\
\hline
\end{tabular}

${ }^{1} T \& O$ - tandem and ovoid applicator; ${ }^{2} T \& C$ - tandem and cylinder applicator; ${ }^{3} \mathrm{CC}$ - correlation coefficient; ${ }^{4} p$ value - $p$ value calculated by Pearson's correlation coefficients; ${ }^{5} t$-test - $p$ value calculated by a two sided paired $t$-test; ${ }^{6} D_{100}$-dose to $100 \%$ of the volume; ${ }^{7} D_{90}$ - dose to $90 \%$ of the volume; ${ }^{8} D_{50}-$ dose to $50 \%$ of the volume; ${ }^{9} D_{2 c c}$-dose to $2 \mathrm{cc}$ of the volume; ${ }^{10} D_{1 c c}$-dose to $1 \mathrm{cc}$ of the volume; ${ }^{11} D_{0.1 c c}$-dose to $0.1 \mathrm{cc}$ of the volume

pelvic lymph nodes (Figures 4 and 5). We found a significant positive correlation between the uterine length and the doses to the external iliac node. This observation may have been caused by the location of the tandem, which was closer to the external iliac node region in the case of a long uterine.

Although the apparent pattern in the implant geometry was not established in this study (Figures 2 and 3), we found that $27 \%$ of points B were not located in any pelvic lymph node regions, which tended to be inside of the pelvis from the results of Table 2 . Several studies have reported that Caucasians have a larger pelvic and iliac width than Japanese individuals [20,21]. The dose delivered to the pelvic lymph nodes may vary in relation to the difference in pelvic and iliac width between Japanese and Caucasians patients, although we cannot compare the dose parameters observed in our study with the previous data [11] simply because of the difference in the dose prescription and fraction. Given our results and the dif- ference in pelvic width between Caucasians and Japanese $[20,21]$, we consider that point $B$ for Caucasians may be located relatively more inside the pelvis compared to that for Japanese. Hence, the dose to the pelvic lymph nodes should be estimated according to the anatomical pelvic size in the case of $2 \mathrm{D}$ conventional planning.

When the dose delivered to lymph nodes was analyzed by applicator type (Table 4), the DVH parameters for the tandem and ovoid applicator were higher than the tandem and cylinder applicator overall. Although there were significant differences between the two applicator types for $\mathrm{D}_{100}, \mathrm{D}_{90}$, and $\mathrm{D}_{50}$ of the external iliac nodes and $D_{100}$ and $D_{90}$ of the internal iliac nodes, the differences were smaller than that of the obturator, and $\mathrm{D}_{2 \mathrm{cc}}$ $\mathrm{D}_{1 \mathrm{cc}}$ and $\mathrm{D}_{0.1 \mathrm{cc}}$ of the external iliac nodes and $\mathrm{D}_{50}, \mathrm{D}_{2 \mathrm{cc}}$ $\mathrm{D}_{1 \mathrm{cc}}$ and $\mathrm{D}_{0.1 \mathrm{cc}}$ of the internal iliac nodes did not show significant differences. However, the dose to the obturator nodes was significantly lower by $0.27-0.67$ Gy for the tandem and cylinder applicator than in case of the 
tandem and ovoid applicator when compared by a $t$-test. This observation may have been due to the location of the obturator node region, which was closer to the ovoid applicator than other lymph nodes.

For cervical cancer patients, who have enlarged nodal disease and receive an external-beam boost dose, the dose contribution to the pelvic lymph nodes from brachytherapy is very important. Lee et al. investigated the dose and equivalent dose contribution of HDR brachytherapy to metastatic pelvic lymph nodes in locally advanced cervical cancer patients [22]. They demonstrated that the brachytherapy dose accounted for about $7 \%$ of the total prescription boost dose to the lymph nodes. In addition, Mohamed et al. evaluated the dose delivered to the lymph nodes by brachytherapy and the effect of brachytherapy image-guided optimization on the lymph node dose. They mentioned that brachytherapy contributes a significant dose to the pelvic lymph nodes and should be considered in the evaluation of total lymph node doses. The dose was 4-6 Gy equivalent total dose in 2 Gy fractions $\left(\mathrm{EQD}_{2}\right)$ for the obturator, external iliac nodes, and internal iliac nodes, when two fractions of MRI-guided pulsed-dose-rate (PDR) brachytherapy were delivered 1 week apart and each fraction was 20 -hourly pulses. The brachytherapy plan optimization aimed to deliver a total EQD 2 (EBRT plus brachytherapy) of at least 85 Gy to the high-risk clinical target volume (HR-CTV) $\mathrm{D}_{90}$, and to keep the $\mathrm{D}_{2 \mathrm{cc}}$ for bladder below $90 \mathrm{~Gy}$, and the $\mathrm{D}_{2 \mathrm{cc}}$ of the rectum, sigmoid, and bowel below 70-75 Gy [23]. Bacorro et al. also analyzed the dose delivered to pelvic lymph nodes during brachytherapy. The planning objectives, summing EBRT plus PDR-IGBT in $\mathrm{EQD}_{2}$, consisted of HR-CTV $D_{90} \geq 85 \mathrm{~Gy}$ and the OAR dose constraints were $75 \mathrm{~Gy}, 75 \mathrm{~Gy}$, and $85 \mathrm{~Gy}$ to $\mathrm{D}_{2 \mathrm{cc}}$ of the rectum, sigmoid, and bladder, respectively. They reported brachytherapy contribution to the treatment of pelvic nodes at the level of approximately 5 Gy in the internal iliac, external iliac, and obturator nodes [24]. Chua et al. quantified the brachytherapy dose contribution to pelvic node groups using the Manchester method. They indicated that the mean cumulative $\mathrm{EQD}_{2}$ doses to the external iliac, the internal iliac, and the obturator nodes were $4.30 \mathrm{~Gy}, 5.95 \mathrm{~Gy}$, and 7.09 Gy, respectively, in patients who received $6 \mathrm{~Gy}$ $\times 4$ fractions [25]. Thus, the brachytherapy dose was reported to make a significant contribution in these studies, and the accurate evaluation of the dose delivered to the pelvic lymph nodes by brachytherapy is essential.

In this simulation study, we adopted only the standard loading pattern [18], and source dwell times were not optimized to minimize the dose to the OAR and maximize the coverage of the HR-CTV, because we used the previous conventional 2D plan data; this is a limitation of the present study. Three-dimensional IGBT was developed to ensure target coverage and avoid exposure to OAR $[12,13]$. However, in low-income countries, where the incidence of cervical cancer is high, 3D imaging is generally not available, and a non-optimized 2D plan is used instead $[26,27,28]$. Therefore, we consider that the findings of this study are important for resource-limited institutes that use the 2D plan.

\section{Conclusions}

Our assessment indicated that the point B dose was a poor surrogate for the dose delivered to the pelvic lymph nodes. Thus, 3D evaluation of the dose delivered to the contoured pelvic lymph nodes is more accurate than single-point estimation using point $B$.

\section{Disclosure}

Authors report no conflict of interest.

\section{References}

1. Barillot I, Horiot JC, Pigneux J et al. Carcinoma of the intact uterine cervix treated with radiotherapy alone: A French cooperative study: Update and multivariate analysis of prognostics factors. Int J Radiat Oncol Biol Phys 1997; 38: 969-978.

2. Bachaud JM, Fu RC, Delannes M et al. Non-randomized comparative study of irradiation alone or in combination with surgery in stage Ib, IIa and "proximal" IIb carcinoma of the cervix. Radiother Oncol 1991; 22: 104-110.

3. Eifel PJ, Winter K, Morris M et al. Pelvic irradiation with concurrent chemotherapy versus pelvic and para-aortic irradiation for high-risk cervical cancer: An update of Radiation Therapy Oncology Group Trial (RTOG) 90-01. J Clin Oncol 2004; 22: 872-880.

4. Morris M, Eifel PJ, Lu J et al. Pelvic radiation with concurrent chemotherapy compared with pelvic and para-aortic radiation for high-risk cervical cancer. N Engl J Med 1999; 340: 1137-1143.

5. Rotman M, Choi K, Guse C et al. Prophylactic irradiation of the para-aortic lymph node chain in stage IIb and bulky stage Ib carcinoma of the cervix, initial treatment results of RTOG 7920. Int J Radiat Oncol Biol Phys 1990; 19: 513-521.

6. Landoni F, Maneo A, Colombo A et al. Randomised study of radical surgery versus radiotherapy for stage Ib-IIa cervical cancer. Lancet 1997; 350: 535-540.

7. Guedea F, Venselaar J, Hoskin P et al. Patterns of care for brachytherapy in Europe: Updated results. Radiother Oncol 2010; 97: 514-520.

8. Perez CA, Breaux S, Madoc-Jones $\mathrm{H}$ et al. Radiation therapy alone in the treatment of carcinoma of uterine cervix I. Analysis of tumor recurrence. Cancer 1983; 51: 1393-1402.

9. Eifel PJ, Thoms WW, Smith TL et al. The relationship between brachytherapy dose and outcome in patients with bulky endocervical tumors treated with radiation alone. Int J Radiat Oncol Biol Phys 1993; 28: 113-118.

10. Ogawa $Y$, Nemoto K, Kakuto $Y$ et al. Results of radiation therapy for uterine cervical cancer using high dose rate remote after loading system. Tohoku J Exp Med 2003; 199: 229-238.

11. Lee LJ, Sadow CA, Russell A et al. Correlation of point B and lymph node dose in 3D-planned high-dose-rate cervical cancer brachytherapy. Int J Radiat Oncol Biol Phys 2009; 75: 803-809.

12. Haie-Meder C, Pötter R, Van Limbergen E et al. Recommendations from Gynaecological (GYN) GEC-ESTRO Working Group (I): Concepts and terms in 3D image-based 3D treatment planning in cervix cancer brachytherapy with emphasis on MRI assessment of GTV and CTV. Radiother Oncol 2005; 74: 235-245.

13. Pötter R, Haie-Meder C, Van Limbergen E et al. Recommendations from gynaecological (GYN) GEC ESTRO working group (II): Concepts and terms in 3D image-based treatment planning in cervix cancer brachytherapy-3D dose volume parameters and aspects of 3D image-based anatomy, radiation physics, radiobiology. Radiother Oncol 2006; 78: 67-77. 
14. Charra-Brunaud C, Harter V, Delannes M et al. Impact of 3D image-based PDR brachytherapy on outcome of patients treated for cervix carcinoma in France: Results of the French STIC prospective study. Radiother Oncol 2012; 103: 305-313.

15. Derks K, Steenhuijsen JLG, Van den Berg HA et al. Impact of brachytherapy technique (2D versus $3 \mathrm{D}$ ) on outcome following radiotherapy of cervical cancer. J Contemp Brachytherapy 2018; 10: 17-25.

16. Paul AG, Nalichowski A, Abrams J et al. Dosimetric evaluation of Point $A$ and volume-based high-dose-rate plans: a single institution study on adaptive brachytherapy planning for cervical cancer. J Contemp Brachytherapy 2018; 10: 202-210.

17. Gebara WJ, Weeks KJ, Jones EL et al. Carcinoma of the uterine cervix: A 3D-CT analysis of dose to the internal, external and common iliac nodes in tandem and ovoid applications. Radiother Oncol 2000; 56: 43-48.

18. Teshima $T$, Inoue $T$, Inoue $T$ et al. High-dose rate intracavitary therapy for cervical cancer with a microSelectron: a preliminary report. Radiat Med 1993; 11: 237-241.

19. Toita T, Ohno T, Kaneyasu Y et al. A consensus-based guideline defining the clinical target volume for pelvic lymph nodes in external beam radiotherapy for uterine cervical cancer. Jpn J Clin Oncol 2010; 40: 456-463.

20. Kimura K. Sex differences of the hip bone among several populations. Okajimas Folia Anat Jpn 1982; 58: 265-275.

21. Hoaglund FT, Shiba R, Newberg AH et al. Diseases of the hip: A comparative study of Japanese oriental and American white patients. J Bone Joint Surg Am 1985; 67: 1376-1383.

22. Lee YC, Rash DL, Stern RL et al. The equivalent dose contribution from high-dose-rate brachytherapy to positive pelvic lymph nodes in locally advanced cervical cancer. Brachytherapy 2013; 12: 555-559.

23. Mohamed SM, Aagaard T, Fokdal LU et al. Assessment of radiation doses to the para-aortic, pelvic, and inguinal lymph nodes delivered by image-guided adaptive brachytherapy in locally advanced cervical cancer. Brachytherapy 2015; 14: 56-61.

24. Bacorro W, Dumas I, Levy A et al. Contribution of image-guided adaptive brachytherapy to pelvic nodes treatment in locally advanced cervical cancer. Brachytherapy 2017; 16: 366-372.

25. Chua GWY, Foo YW, Tay GH et al. Assessing dose contribution to pelvic lymph nodes in intracavitary brachytherapy for cervical cancer. J Contemp Brachytherapy 2017; 9: 345-353.

26. Suneja G, Brown D, Chang A et al. American Brachytherapy Society: Brachytherapy treatment recommendations for locally advanced cervix cancer for low-income and middle-income countries. Brachytherapy 2017; 16: 85-94.

27. LaVigne AW, Triedman SA, Randall TC et al. Cervical cancer in low- and middle-income countries: Addressing barriers to radiotherapy delivery. Gynecol Oncol Rep 2017; 22: 16-20.

28. Carvalho Hde A, Mendez LC, Stuart SR et al. Implementation of image-guided brachytherapy (IGBT) for patients with uterine cervix cancer: A tumor volume kinetics approach. J Contemp Brachytherapy 2016; 8: 301-307. 